\title{
Cloud Computing Issues and Countermeasures
}

\author{
Nazish Fatima \\ Computer Science \& Software \\ Engineering Department \\ University of Hai'l \\ Hai'l, KSA
}

\author{
Zahida Parveen \\ Computer Science \& Software \\ Engineering Department \\ University of Hai'l \\ Hai'l, KSA
}

\begin{abstract}
Cloud computing is an arrangement of Information Technology administrations offered to clients over the web on a leased base. Such administrations empower the associations to scale-up or downsize their in-house establishments. By and large, cloud administrations are given by an outside supplier who has the game plan. Cloud computing has numerous focal points, for example, adaptability, productivity, versatility, combination, and capital lessening. In addition, it gives a progressed virtual space for associations to convey their applications or run their operations. With dismissal to the conceivable advantages of Cloud computing administrations, the associations are hesitant to put resources into Cloud computing chiefly because of security concerns. Security in Cloud computing is an essential and basic angling that upset the development of Cloud computing, and has various issues and issue identified with it. Cloud administration supplier and the cloud administration purchaser ought to ensure that the cloud is sufficiently sheltered from all the outer dangers so that the client does not confront any issue, for example, loss of information or information burglary. There is likewise a probability where a noxious client can infiltrate the cloud by mimicking a real client, along these lines tainting the whole cloud and influences numerous clients who are sharing the contaminated cloud. This paper firstly records the parameters that influence the security of the cloud then it investigates the cloud security issues and issues confronted by cloud administration supplier and cloud administration customer, for example, information, protection, and contaminated application and security issues. It likewise plates a few tips for handling these issues.
\end{abstract}

Keywords- Cloud computing; issues; security; parameters

\section{INTRODUCTION}

A couple of years prior, unique states of cloud were utilized to mean the web and the internet. A while later the cloud has been used to speak to a more particular thought, which is the Cloud Computing. The extension and development of the electronic administrations requires constant change as far as foundation. Cloud computing offers a moderately minimal effort adaptable other option to inhouse infrastructure, both in equipment and programming [1]. The fundamental thought of cloud computing is to convey both programming and equipment as administrations .People and associations have been thinking about administrations over the cloud to cut the expenses of use, with no pay in using late advances. Nevertheless, using administrations over the cloud is gone with numerous questions generally about security issues.

\section{ISSUES IN CLOUd COMPUTING}

This paper discusses issues of cloud computing with respect to technique and also services.

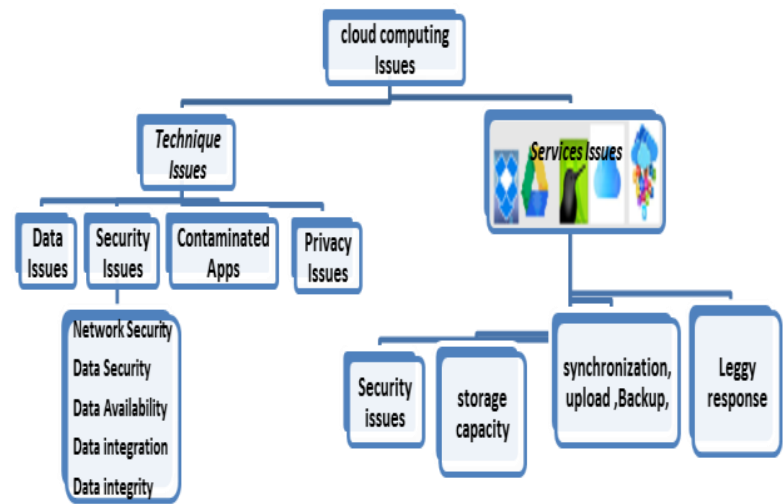

Figure 1. Cloud computing issues

\section{A. Issues In Cloud Computing Technique}

The cloud administration supplier for cloud ensures that the client does not confront any issue, for example, loss of information or information robbery. There is likewise plausibility where a noxious client can enter the cloud by imitating a true blue client, there by contaminating the whole cloud [6]. This prompts influences numerous clients who are sharing the tainted cloud. There are four sorts of issues raise while talking about security of a cloud [7].

1. Data Issues

2. Privacy issues

3. Contaminated Application

4. Security issues

Data Issues: touchy information in a distributed computing environment rise as real issues with respect to security in a cloud based framework. Firstly, at whatever point information is on a cloud, anybody from anyplace 
whenever can get to information from the cloud since information might be regular, private and touchy information in a cloud. So in the meantime, numerous distributed computing administration purchaser and supplier get to and alter information. Consequently there is a need of a few information uprightness techniques in distributed computing. Also, information taking is a one of major issue in a distributed computing environment. Numerous cloud administration suppliers don't give their own particular server rather they procure server from other administration suppliers because of it is cost emotional and adaptable for operation and cloud supplier. So there is a much likelihood of information can be stolen from the outer server. Thirdly, Data misfortune is a typical issue in distributed computing. In the event that the distributed computing administration supplier close down his administrations due some money related or lawful issue then there will be lost information for the client. Additionally, information can be lost or harm or ruined because of miss event, normal catastrophe, and flame. Due to above condition, information may not be accesses able to clients. Fourthly, information area is one of the issues what requires center in a distributed computing environment[4]. Physical area of information stockpiling is vital and vital. It ought to be straightforward to client and client. Merchant does not uncover where every one of the information's are put away.

Privacy Issues: The cloud figuring organization supplier must guarantee that the customer singular information is particularly secured from various suppliers, and customer. As most of the servers are outside, the cloud organization supplier should guarantee who is getting to the data and who is keeping up the server with the objective that it enables the supplier to secure the customer's near and dear information.

Contaminated Application: cloud processing organization supplier should have the finish access to the server with all rights with the ultimate objective of checking and upkeep of server. So this will keep any vindictive customer from exchanging any defiled application onto the cloud which will truly impact the customer and dispersed processing organization.

Security issues: cloud computing security must be done on two levels. One is on supplier level and another is on the customer level. Circulated processing organization supplier should guarantee that the server is particularly secured from all the outside perils it may go over [10]. Regardless of the way that the circulated processing organization supplier has given a better than average security layer to the customer and customer, the customer should guarantee that there should not be any loss of data or take or adjusting of data for various customers who are using the same cloud due to its movement. A cloud is awesome exactly when there is a fair security gave by the organization supplier to the customer [11].

\section{B. Parameters Influencing Cloud Security}

There are different security issues in cloud computing as it conceals various advances including systems, databases, working structures, virtualization, resource booking, the trade organization, stack modifying, concurrent control and memory administration[9]. The accompanying graph demonstrates the parameters that impact cloud security.

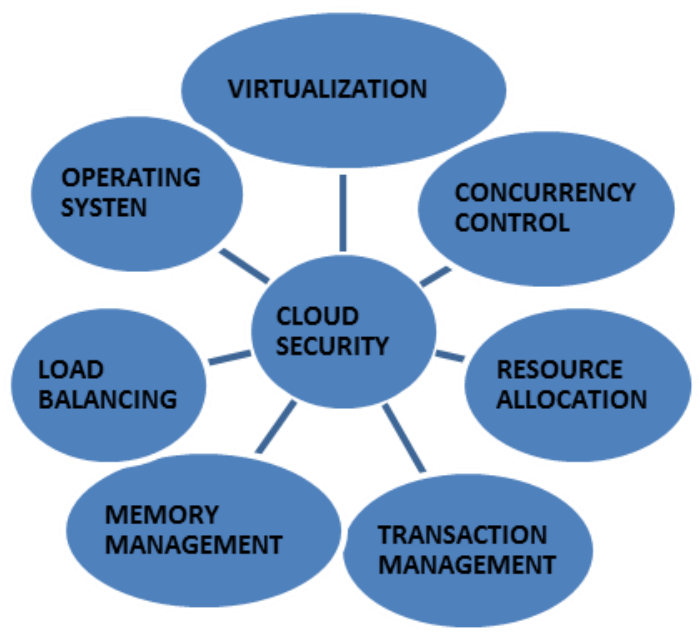

Figure 2. Parameters that influenced clouds security

Security issues for countless structures and headways are material to distributed computing [3]. For example, the framework that interconnects the systems in a cloud must be secure. In addition, virtualization perspective in distributed computing brings about a couple security concerns. Case in point, mapping the virtual machines to the physical machines must be finished securely. Data security incorporates encoding the data and furthermore ensuring that fitting game plans are actualized for data sharing. In like manner, resource parcel and memory organization figuring's must be secure. Finally, information mining strategies may be germane to malware acknowledgment in mists.

Cloud Computing Security major Issues are described briefly below [12].

\section{1) Access to Servers \& Applications}

In cloud administrative access steered via Internet that is the main security threat which is intern related to security policies.

\section{2) Data Transmission}

In processing time more than $70 \%$ data is not encrypted, this unencrypted data is a serious threat to secure data transmission.

\section{3) Virtual Machine Security}

The dynamic nature of Virtual Machines and the possibility of making the clones and copies is a big hurdle in maintaining consistency and security. 


\section{4) Network Security}

In cloud the network security problem is consist of reused IP address, attacks on DNS and attacks of different kinds of Sniffers.

\section{5) Data Security}

The enterprise data is warehoused out of the boundary of the enterprise at Service provider premises. Therefore, the service provider must implement added security checks to guarantee data security.

\section{6) Data Privacy}

Another key concern in cloud security is privacy as all the Data in the cloud is distributed globally that open the concerns about influence, privacy and data exposure

\section{7) Data Integrity}

Clouds usually store the generated data in cloud computing, this method open the way for lose control over data from users point of view.it is also a fact that Data sleaze can happen with any type of media and at any level of storage.

\section{8) Data Location}

Data location is again a big issue in concern with security as there are many users and huge type of data that need to be available locally within the user promises, as in cloud user is not aware of the exact location of their data so it can also create problem.

\section{9) Data Availability:}

Data Availability is one of the major apprehensions of undertaking and security precarious organizations. When storing data at distant systems, users can suffer from system failures. Data will not be available when cloud stops working.

\section{0) Data Segregation}

The storage is shared by the data from different users and as discussed earlier the encryption is not easy to implement in cloud so there arises a big threat of data Segregation.

\section{1) Security Policy and Compliance}

The cloud services provider companies need to be have a clear and strict policies for security provision to the end user. Enterprises need to demonstrate acquiescence with security standards.

\section{2) Patch management}

Patch management is a type of systems management that includes getting, checking, and installing patches (code changes) to a controlled computer system. In cloud patch management is done on user promises so open the risk of security.

\section{Issues In Cloud Computing Services}

Here we will discuss about the major services of cloud computing having some major problems. A survey report of specialized bolster site Fix Ya demonstrates that clients of the main five cloud storage services are most worried about limit, security and missing documents. FixYa got some information about the main five issues cloud storage clients have with Dropbox, Google Drive, Sugar Sync, iCloud and Box.[5]

\section{Dropbox 8}

Dropbox is a standout amongst the most famous storage service administrations. FixYa reviewed about Dropbox and picked the following concerns which are mentioned in the fig-3 below .FixYa clients who depend on Dropbox for individual substance, for example, photographs, music or amusement, aren't disheartened by the security issues, Fix Ya noted.

"Notwithstanding, individual clients using Dropbox to store individual money related data or comparative things that would represent a security hazard ought to stay away," FixYa said. "The same goes for medium sized organizations searching for a simple approach to share bank explanations or exclusive business data."

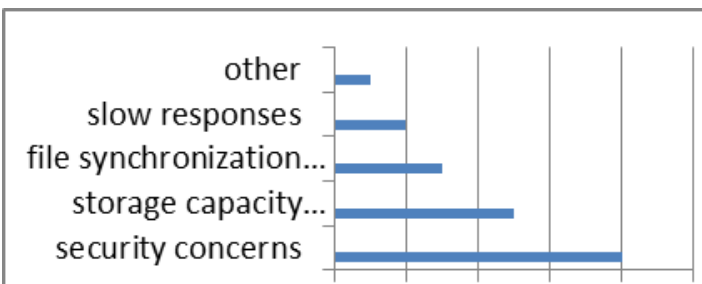

$0 \% \quad 10 \% \quad 20 \% 30 \% \quad 40 \% 50 \%$

Figure 3. Issues in Dropbox Service

\section{Google Drive}

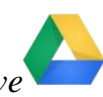

When it came to Google Drive, missing, issues with synchronizing records, automatic conversion unknown errors are major issues of the customers of Google drive. "Despite the fact that security concerns are not as conspicuous amongst Fix Ya clients contrasted with other cloud gadgets, Drive still encounters some broad ease of use issues that should be tended to," FixYa said. "Generally, be that as it may, these issues can be understood rapidly. "See fig-4 below.

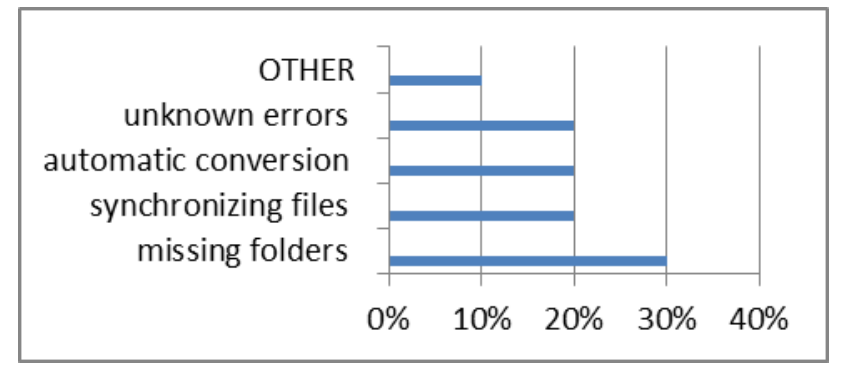

Figure 4. Issues in Google Drive Service 


\section{SugarSync if}

The absence of QuickBooks bolster finished the worries clients had with Sugar Sync. QuickBooks is a business bookkeeping programming for organizations .Since Sugar Sync doesn't bolster QuickBooks; it is an extreme offer for monetary clients. Some of storage ,file syncing,iTune file problem. Here is the brief issues of SugarSync in Fig. 5.

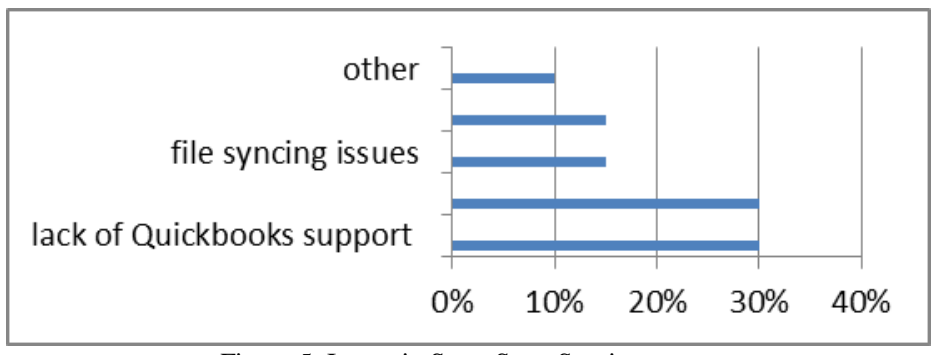

Figure 5. Issues in SugarSync Service

\section{IV. iCloud}

Apple's iCloud storage administration took a hit after clients overhauled to OS X Mountain Lion, as indicated in the survey by FixYa. "Whether it be agreeing to the administration, synchronizing the "Notes" application between gadgets, or moving down records and different applications, iCloud on the new Mountain Lion is not impeccable by any methods." FixYa prescribed that clients encountering issues with matching up Notes attempt Evernote. Moreover, the powerlessness to adjust documents with non-Apple gadgets in iCloud was noticed in survey. other file syncing ,note syncing and security issues are measured in Fig. 6.

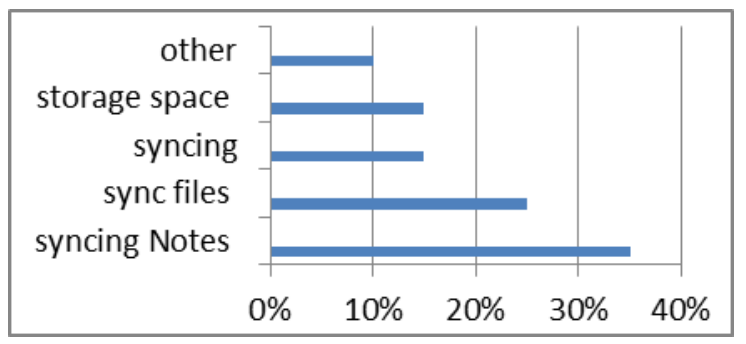

Figure 6. Issues in icloud Service

V. Box

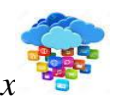

Box, a cloud storage benefit that is most well-known with little organizations due to its local security abilities and administration components, was pounded on the issue of security and transferring issues. A quarter century of overview respondents picked "security concerns"; another picked "transfer issues." While security issues don't seem, by all accounts, to be an issue for extensive scale business accounts, Fix Ya clients have reported worries with security for their free individual records". While Fix Ya can't take care of the security worries with Box, it recommended that clients experiencing difficulty transferring documents ought to restart their PCs and clear the program store before attempting again. Also leggy response and upload issues were noticed with the following ratio in Fig. 7.

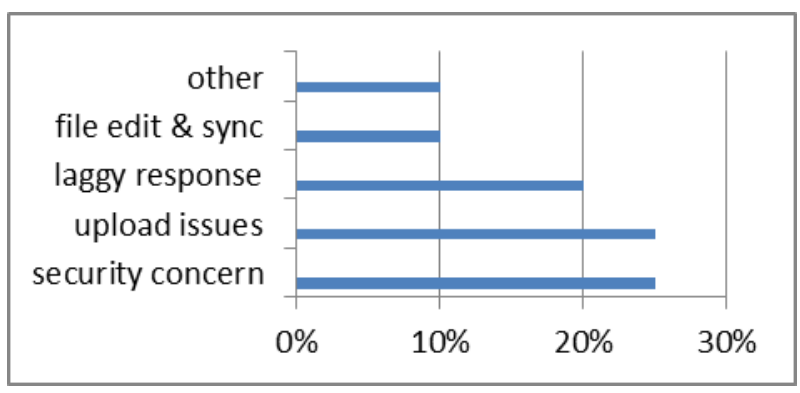

Figure 7. Issues in Box Service

In cloud the issues in services point of view as discussed above one can easily conclude that each service has its on issues and concerns the whole findings can be summarize in the following given table 1.1.

TABLE 1.1: SUMMARY OF CLOUD SERVICES ISSUES

\begin{tabular}{|c|c|c|}
\hline SERVICE & PEAK Issue & $\%$ \\
\hline DrOP BOX & SECURITY & $40 \%$ \\
\hline $\begin{array}{l}\text { GOOGLE } \\
\text { DRIVE }\end{array}$ & $\begin{array}{l}\text { MISSING } \\
\text { FOLDERS }\end{array}$ & $30 \%$ \\
\hline SUGAR SYNC & STORAGE SPACE & $30 \%$ \\
\hline I Cloud & Syncing Note & $35 \%$ \\
\hline Box & SECURITY & $25 \%$ \\
\hline
\end{tabular}

\section{Countermeasures For Cloud Issues}

There are a few tips and traps that cloud security arrangement suppliers ought to remembered when they conveys their support of cloud administration buyer in an open cloud arrangement[2].

Confirm the entrance controls: Set up data to get control with rights and after that check these passage controls by the cloud organization supplier at whatever point data is being used by cloud organization client. To complete get to control methods for client side, the cloud organization supplier must depict and assurance that the principle endorsed customers can get to the customer or buyer's data.

Control the customer access gadgets: Be sure the buyer's passage devices or centers, for instance, Personal Computers, 
virtual terminals, papers, handouts and cell phones are adequately secure. The loss of an endpoint get to contraption or access to the device by an unapproved customer can scratch off even the best security traditions in the cloud. Make certain the customer handling contraptions are directed fittingly and secured from malware working and supporting impelled acceptance highlights.

Screen the Data Access: cloud organization suppliers need to ensure about whom, when and what data is being gotten to for what reason. Case in point various site or server had a security protestation regarding snooping practices by various people, for instance, listening to voice calls, scrutinizing messages and individual data etcetera.

Offer requested records and Verify the information cancellation: If the customer or customer needs to report its consistence, then the cloud organization supplier will share charts or whatever other information or give audit records to the buyer or customer. Furthermore affirm the most ideal deletion of data from shared or reused devices. Various suppliers don't suit the most ideal degaussing of data from drives each time the drive space is left. Request an ensured cancelation get ready and have that method made into the assertions.

Security check occasions: Ensure that the cloud organization supplier gives enough bits of knowledge about fulfillment of ensures, break remediation and reporting probability. These security events will portray commitment, certifications and exercises of the circulated figuring organization supplier.

\section{CONCLUSION}

Cloud computing has been a surpassing shift so far in terms of using the present innovations. The pattern of having cloud administrations as a feature of an association appears to be increasing more significance. Particularly in this time the cycle of presenting more mechanical advancements is getting shorter. For some reasons, including the decrease of capital consumptions, associations need to consider using cloud administrations as a fundamental piece of their establishments. All things considered, different difficulties are precluding the accomplishment of tremendous arrangement and acknowledgment levels. The principle disadvantage of the current cloud administration executions is their powerlessness to give an authorized high-security level. In addition, security certification requirements to cover the transmission channels which may incorporate an outsider. Both the cloud administration supplier and the client ought to ensure that the cloud is sufficiently protected from all the outer dangers, so there will be a solid and common comprehension between the client and the cloud administration supplier. The biggest crevices between cloudsecurity practice and cloud-security research hypothesis lie in the way that the suppositions in the exploration forget some imperative contrasts between real cloud security and virtual machine security. Examination ought to focus on these crevices and contrasts and its evacuation. One of the bits of the structure may build up an approach to screen the cloud's administration programming, and another may be an advancement of segregated preparing for particular customers' applications. Individuals' conduct can be followed and checked for case whether individuals let the robotic fixing programming to run, or redesigning hostile to infection programming definitions, or whether individuals see how to solidify their virtual machines in the cloud.

\section{REFERENCES}

[1] M. Armbrust, A. Fox, R. Grith, A. D. Joseph, R. Katz, A.Konwinski, G. Lee, D. Patterson, A. Rabkin, I. Stoica andM. Zaharia, "A View of Cloud Computing," Communicationsof the ACM, Vol. 53, No.4,2010,pp50-58.http://dx.doi.org/10.1145/1721654.1721672

[2] A. M. Andrew, "Cloud Computing: Views on Cybersyn,"Kybernetes, Vol. 41, No. 9, 2012, pp. 13961399.http://dx.doi.org/10.1108/03684921211275450

[3] S. Dhar, "From Outsourcing to Cloud Computing: Evolutionof It Services," Management Research Review, Vol.35, No. 8, 2012, pp. 664-675.http://dx.doi.org/10.1108/01409171211247677

[4] S. Subashini and V. Kavitha, "A Survey on Security Issuesin Service Delivery Models of Cloud Computing,"Journal of Network and Computer Applications, Vol. 34,No. 1, 2011, pp. 1-11.

[5] http://www.sciencedirect.com/science/article/pii/S108480

[6] http://www.computerworld.com/article/2493144/cloudcomputing/the-top-5-issues-with-the-top-5-cloud-storageservices.html.

[7] S. Ramgovind, M. Elo and E. Smith, "The Managementof Security in Cloud Computing," Information Securityfor South Africa, Sandton, 24 August 2010, pp. 1-7.International Conference on Cloud Computing, Bangalore,21-25 September 2009, pp. 109-11

[8] P. Mell and T. Grance, "The NIST Definition of CloudComputing," Computer Security Division, IT Laboratory,National Institute of Standards and Technology, Gaithersburg,2011.

[9] http://csrc.nist.gov/publications/nistpubs/800-145/SP800-145.pdf

[10] Tackle your client's security issues with cloud computing in 10 steps, http://searchsecuritychannel.techtarget.com/tip/Tackle-yourclients-security-issues-with-cloud-computing-in-10-steps.

[11] ProblemsFacedbyCloudComputing,LordCrusAd3r,dl.packetstormsecu rity.net/.../ProblemsFacedbyCloudComputing.pdf.

[12] KevinHamlen,MuratKantarcioglu,LatifurKhan,BhavaniThuraisingha $\mathrm{m}$,SecurityIssuesforCloudComputing,InternationalJournalofInformatio nSecurityandPrivacy,4(2),39-51,UniversityofTexas,USA,AprilJune2010.

[13] Dr.GurdevSingh,ShanuSood,AmitSharma,"CM-Measurement FacetsforCloudPerformance",IJCA,,Lecturer,Computerscience\&Engin eering,EternalUniversity,BaruSahib(India), Volume23No.3,June2011.

[14] IRACST - International Journal of Computer Science and Information Technology \& Security (IJCSITS) Vol. 1, No. 2, December 2011

\section{AUTHORS PROFILE}

Nazish Fatima is M.Sc. in Computer Science (MSCS) fellow of Virtual University of Pakistan. She has completed Master in Computer Science (MCS) from University of Karachi and Master in Islamic Studies with Computer Technology from Sheikh Zayed Islamic Centre, University of Karachi, Pakistan. She passed PGD (Post Graduate Diploma) in Mathematics from University of Karachi Pakistan. She has eleven years of teaching experience at graduate and post graduate levels courses, currently working as lecture of computer science in University of Ha'il, Saudi Arabia form year 2010. Her 
research interest includes cloud computing, network security, mobile and pervasive computing and big data.

Zahida Parveen has completed MCS (Master in Computer Science)

NUML Islamabad, Islamabad, Pakistan. B.Sc (Computer) Punjab University, Punjab, Pakistan. She is currently working as lecturer of computer science in University of Ha'il from year 2010. Her research interests are Cloud Computing, Programming Languages, Software Engineering and Big Data.

(C) 2017 by the author(s); licensee Empirical Research Press Ltd. United Kingdom. This is an open access article distributed under the terms and conditions of the Creative Commons by Attribution (CC-BY) license. (http://creativecommons.org/licenses/by/4.0/). 